\title{
Long term results of cardioverter-defibrillator implantation in patients with right ventricular dysplasia and malignant ventricular tachyarrhythmias
}

\author{
R Tavernier, S Gevaert, J De Sutter, A De Clercq, H Rottiers, L Jordaens, W Fonteyne
}

\begin{abstract}
Objective-To study the outcome of patients with arrhythmogenic right ventricular dysplasia treated with an implantable cardioverter-defibrillator (ICD) for ventricular tachyarrhythmias complicated by haemodynamic collapse.

Design-Observational study.

Setting-University hospital.

Patients-Nine consecutive patients (eight male, one female; mean (SD) age, 36 (18) years) with arrhythmogenic right ventricular dysplasia presenting with ventricular tachycardia and haemodynamic collapse $(n=6)$ or ventricular fibrillation $(n=3)$, treated with an ICD.

Main outcome measures-Survival; numbers of and reasons for appropriate and inappropriate ICD interventions.

Results-After a mean (SD) follow up of 32 (24) months, all patients were alive. Six patients received a median of 19 (range 2-306) appropriate ICD interventions for events detected in the ventricular tachycardia window; four received a median of 2 (range 1-19) appropriate ICD interventions for events detected in the ventricular fibrillation window. Inappropriate interventions were seen for sinus tachycardia (18 episodes in three patients), atrial fibrillation (three episodes in one patient), and for non-sustained polymorphic ventricular tachycardia (one episode in one patient).

Conclusions-Patients with arrhythmogenic right ventricular dysplasia and malignant ventricular arrhythmias have a high recurrence rate requiring appropriate ICD interventions, but they also often have inappropriate interventions. Programming the device is difficult because this population develops supraventricular and ventricular tachyarrhythmias with similar rates.

(Heart 2001;85:53-56)
\end{abstract}

Keywords: arrhythmogenic right ventricular dysplasia; implantable cardioverter defibrillator; arrhythmia

Department of Cardiology, University

Hospital Gent, De

Pintelaan 185, 9000

Gent, Belgium

R Tavernier

S Gevaert

J De Sutter

A De Clercq

H Rottiers

L Jordaens

W Fonteyne

Correspondence to: Dr Tavernier

rene.tavernier@rug.ac.be

Accepted 12 September 2000
Arrhythmogenic right ventricular dysplasia is an idiopathic cardiomyopathy characterised by adipose or fibroadipose substitution in the right ventricle combined with the occurrence of ventricular arrhythmias of right ventricular origin. The disease can be familial, with a autosomal dominant inheritance. Patients usually present between the ages of 20 and 50 years with palpitations, chest discomfort, and syncope or near syncope. Most may be treated safely with class III antiarrhythmic agents. ${ }^{1}$ However, some patients present with sudden cardiac death or sustained ventricular tachycardia with haemodynamic collapse. Implantation of a cardioverter-defibrillator (ICD) in these patients has been suggested as a treatment option, but data on long term follow up of such patients are limited. ${ }^{2-4}$ We report the outcome in nine patients with arrhythmogenic right ventricular dysplasia and malignant ventricular arrhythmias treated with an ICD.

\section{Methods}

PATIENT POPULATION

A single chamber cardioverter defibrillator was implanted in nine consecutive patients (eight male, one female; mean (SD) age, 36 (18) years, range 11-63) with arrhythmogenic right ventricular dysplasia, who presented with ventricular tachycardia and haemodynamic collapse $(n=6)$ or ventricular fibrillation $(n=3)$ (table 1). In seven patients the presenting

Table 1 Patient characteristics

\begin{tabular}{|c|c|c|c|c|c|c|c|}
\hline Patient & $\operatorname{Sex}$ & $\begin{array}{l}\text { Age } \\
\text { (years) }\end{array}$ & $\begin{array}{l}\text { Presenting arrhythmia } \\
\text { (CL in ms) }\end{array}$ & Circumstances & $\begin{array}{l}\text { LVEF } \\
(\%)\end{array}$ & AAR before implant & $A A R$ after implant \\
\hline 1 & Male & 54 & VT (295 ms) & Rest & 59 & Amio, flec, mex, sot & Sot \\
\hline 2 & Male & 11 & $\mathrm{VT}(260 \mathrm{~ms})$ & Exercise & 39 & Sot & Sot \\
\hline 3 & Male & 54 & VT (270 ms) & Rest & 42 & Amio, prop, phen, sot & Prop, phen, cel \\
\hline 4 & Male & 34 & $\mathrm{VT}(250 \mathrm{~ms})$ & Exercise & 61 & Amio & Sot \\
\hline 5 & Female & 19 & VF & Exercise & 71 & - & Sot, met \\
\hline 6 & Male & 34 & VF & Exercise & 69 & - & Sot, amio \\
\hline 7 & Male & 30 & $\mathrm{VF}$ & Exercise & 63 & - & Sot \\
\hline 8 & Male & 63 & VT (280 ms) & Exercise & 62 & - & - \\
\hline 9 & Male & 22 & VT (270 ms) & Exercise & 72 & Sot & Sot \\
\hline
\end{tabular}

AAR, antiarrhythmic drugs; amio, amiodarone; cel, celiprolol; CL, cycle length; flec, flecainide; LVEF, left ventricular ejection fraction; met, metoprolol; mex, mexiletine; phen, phenytoin; prop, propafenone; sot, sotalol; VT, ventricular tachycardia; VF, ventricular fibrillation. 


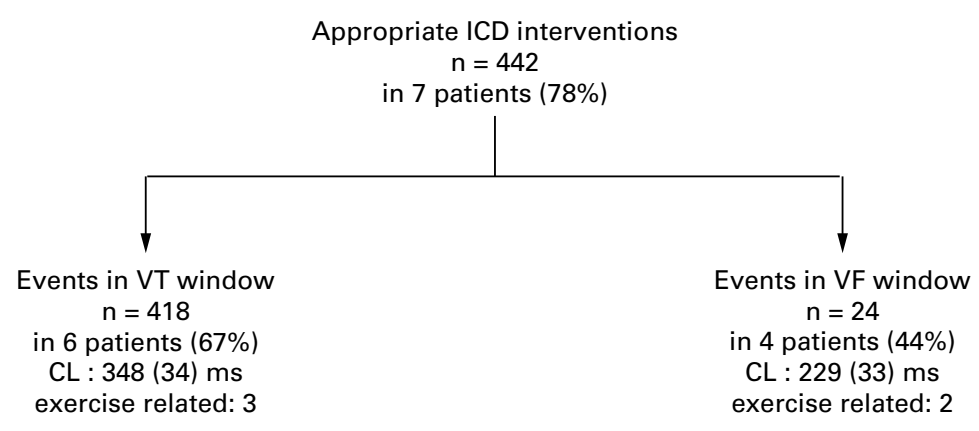

Figure 1 Characteristics of appropriate implantable cardioverter-defibrillator (ICD) interventions. CL, cycle length; VF, ventricular fibrillation; VT, ventricular tachycardia.

arrhythmia occurred during exercise. The diagnosis of arrhythmogenic right ventricular dysplasia was based upon the criteria published by the task force of the working group on myocardial and pericardial disease of the European Society of Cardiology and the scientific council of cardiomyopathies of the International Society and Federation of Cardiology. ${ }^{5}$ Five patients presenting with ventricular tachycardia had recurrent ventricular arrhythmias or their arrhythmias remained inducible despite antiarrhythmic drugs $(n=5)$ or ablation $(n=3)$ before ICD implantation. Patients presenting with ventricular fibrillation were treated with a defibrillator without a trial of antiarrhythmic drugs or ablation. After ICD implantation all but one patient received additional antiarrhythmic drugs. Sotalol was given in seven patients for the prevention of recurrence of ventricular arrhythmias and to limit the maximum heart rate during exercise. In patient 6 , sotalol was changed to amiodarone because of recurrent atrial fibrillation. In patient 5, sotalol was changed to metoprolol to avoid $T$ wave sensing. Patient 3 received celiprolol, propafenone, and phenytoin because this combination was the most effective at preventing recurrences of ventricular tachycardia before ICD implantation. Patient 8 refused any drug treatment.

CARDIOVERTER-DEFIBRILLATOR IMPLANTATION AND PROGRAMMING

A single chamber ICD was implanted in all the patients. Programming of the ventricular tachycardia detection window (mean (SD) cycle length 339 (20) ms) was based upon the cycle length of the clinical (271 (16) ms) or induced (315 (43) ms) ventricular tachycardia. Events detected in the ventricular tachycardia window were treated with antitachycardia pacing, followed by cardioversion if pacing was unsuccessful in terminating the arrhythmia. In two patients presenting with ventricular fibrillation (patients 5 and 6), no ventricular tachycardia detection was programmed because no ventricular arrhythmia could be induced during the electrophysiological study. The sudden onset algorithm was not used because seven of the nine patients had exercise induced ventricular tachycardia. A rate stability of $50 \mathrm{~ms}$ was programmed in a single patient who had a history of atrial fibrillation. In all patients a ventricular fibrillation detection window was programmed, with a mean detection cycle length of 271 (24) ms. Events detected in the ventricular fibrillation window were treated by defibrillation.

FOLLOW UP

All patients were seen one month after implantation and every three months thereafter. In the event of a shock or hospital admission, an additional ICD interrogation was performed. ICD interventions (antitachycardia pacing or shock delivery) were classified as appropriate or inappropriate on the basis of rate behaviour, electrogram morphology, and the result of the intervention. All ICD interventions were reviewed by three investigators (RT, SG, and $\mathrm{ADC}$ ) and classified by consensus.

\section{Results}

After a mean (SD) follow up of 32 (24) months, all patients were alive. One patient underwent heart transplantation after 27 months because of congestive heart failure from progressive involvement of the left ventricle and because of recurrent episodes of ventricular tachycardia. Seven patients had received at least one appropriate ICD intervention. Four patients received at least one inappropriate intervention.

\section{APPROPRIATE ICD INTERVENTIONS}

We registered 442 appropriate interventions in seven patients (78\%) (fig 1). These interventions were for 418 events detected in the ventricular tachycardia detection window in six patients and for 24 events detected in the ventricular fibrillation detection window in four patients (table 2). All patients who presented with ventricular tachycardia experienced recurrences requiring appropriate interventions. Only one patient who presented with ventricular fibrillation had an event appropriately detected in the ventricular tachycardia window.

The mean cycle length of the 418 events in the ventricular tachycardia window was 348 (34) ms. These events were exercise related in three patients. One patient who required heart

Table 2 Appropriate ICD interventions

\begin{tabular}{|c|c|c|c|c|c|c|}
\hline Patient & $\begin{array}{l}\text { Appropriate } \\
\text { interventions }\end{array}$ & $\begin{array}{l}\text { Events in VT window } \\
\text { (n;CL range in ms) }\end{array}$ & $\begin{array}{l}\text { Events in } V T \text { window } \\
\text { treated with } A T P / \text { shock }\end{array}$ & $\begin{array}{l}\text { Events in } V F \text { window } \\
\text { ( } n \text {; CL range in ms) }\end{array}$ & Circumstances & $\begin{array}{l}\text { Follow up } \\
\text { (months) }\end{array}$ \\
\hline 1 & 325 & $306 ; 300$ to 380 & $281 / 25$ & $19 ; 200$ to 280 & Rest & $\star 27$ \\
\hline 2 & 7 & $5 ; 290$ to 340 & $5 / 0$ & $2 ; 260$ to 255 & Exercise & 22 \\
\hline 3 & 34 & $34 ; 290$ to 400 & $33 / 1$ & 0 & Exercise and rest & 18 \\
\hline 4 & 68 & $67 ; 290$ to 350 & $66 / 1$ & $1 ; 230$ & Rest & 87 \\
\hline 7 & 2 & $2 ; 300$ to 310 & $1 / 1$ & 0 & Rest & 19 \\
\hline 8 & 2 & 0 & $0 / 0$ & $2 ; 160$ to 250 & Exercise & 44 \\
\hline 9 & 4 & $4 ; 310$ to 320 & $3 / 1$ & 0 & Exercise & 4 \\
\hline
\end{tabular}

^Heart transplant.

ATP, antitachycardia pacing; CL, cycle length; VT, ventricular tachycardia; VF, ventricular fibrillation. 


\begin{tabular}{l}
\multicolumn{4}{c}{ Inappropriate ICD interventions } \\
$\mathrm{n}=22$ \\
in 4 patients (44\%)
\end{tabular}

$\overline{\mathrm{AF}}$, atrial fibrillation; ATP, antitachycardia pacing; CL, cycle length; NSPVT, non-sustained polymorphic ventricular tachycardia; ST, sinus tachycardia.

transplantation had received 306 interventions for events detected in the ventricular tachycardia window. Antitachycardia pacing terminated the event in $93 \%$ of the cases. Cardioversion was necessary in five patients for 29 events.

The mean cycle length of the 24 events detected in the ventricular fibrillation window was 229 (33) $\mathrm{ms}$ and they were exercise related in two patients. The transplanted patient had received 19 interventions for events detected in the ventricular fibrillation window. All these events were terminated by defibrillation.

\section{INAPPROPRIATE ICD INTERVENTIONS}

We registered 22 inappropriate ICD interventions (16 episodes of antitachycardia pacing and six episodes of shock delivery) in four patients for sinus tachycardia (18 episodes in three patients), atrial fibrillation (three episodes in one patient), and non-sustained polymorphic ventricular tachycardia (one episode in one patient) (fig 2 and table 3 ). The episodes of sinus tachycardia with a cycle length of 328 (18) ms were all exercise related (swimming in one patient and cycling in two patients). They

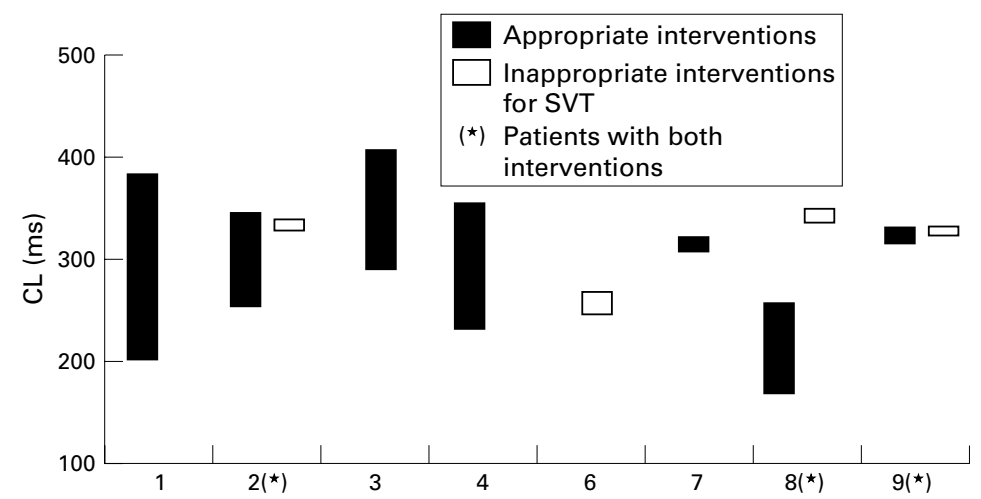

Figure 3 Range of cycle lengths of appropriate and inappropriate implantable cardioverter-defibrillator interventions for supraventricular tachycardias on an individual basis. CL, cycle length; SVT, supraventricular tachycardia; (*) patients with both appropriate and inappropriate interventions. occurred despite treatment with sotalol in two patients. The episode of non-sustained ventricular tachycardia with a cycle length of $160 \mathrm{~ms}$ was exercise related and was detected in the ventricular fibrillation window. Three episodes of atrial fibrillation with a cycle length ranging from $240-260 \mathrm{~ms}$ were exercise related and detected in the ventricular fibrillation window.

\section{Discussion}

In this study we showed that patients with arrhythmogenic right ventricular dysplasia complicated by ventricular tachyarrhythmias resistant to drug treatment or ablation and who are treated with a cardioverter-defibrillator have a high arrhythmia recurrence rate but a good prognosis. This is in agreement with data published by Breithardt and colleagues, who also observed a high recurrence rate of ventricular arrhythmias (50\%) in a similar population of patients with arrhythmogenic right ventricular dysplasia treated with an ICD. ${ }^{2}$ Unfortunately, such patients also suffer from frequent inappropriate interventions because of supraventricular tachycardias (especially sinus tachycardia during exercise) with a fast ventricular response.

APPROPRIATE ICD INTERVENTIONS

Four of our nine patients had events with very short cycle lengths $(\leqslant 260 \mathrm{~ms})$. All these events were potentially life threatening. None of these patients had ventricular fibrillation as the presenting arrhythmia. This observation is in agreement with published data suggesting that patients with arrhythmogenic right ventricular dysplasia and drug refractory sustained ventricular tachycardia have an increased risk of sudden cardiac death. ${ }^{6}$ Three patients had frequent ICD interventions. It is unclear whether this high frequency was related to disease progression. One of these patients showed progressive involvement of the left ventricle requiring heart transplantation, while in the other two no clear evidence of extensive left ventricular involvement was present, as judged by repeated measurements of the left ventricular ejection fraction. Finally, patients with arrhythmogenic right ventricular dysplasia often have exercise related recurrences of their arrhythmias. This may influence the choice and the programming of the ICD (see below).

\section{INAPPROPRIATE ICD INTERVENTIONS}

Despite the availability of different algorithms to avoid inappropriate ICD interventions, these occurred in four of our nine patients. In our overall single chamber ICD population, we have observed inappropriate interventions in $13 \%$ of the patients.

A high rate detection window is important to avoid inappropriate ICD interventions caused by supraventricular tachycardias. ${ }^{8}$ However, the mean lengths of the inappropriate and appropriate interventions were similar (313 (45) v 330 (53) ms, NS), and cycle lengths of ventricular and supraventricular arrhythmias overlapped significantly in two patients (fig 3). This overlap occurred despite 
the administration of sotalol to prevent ventricular tachyarrhythmias and to limit the maximum heart rate during exercise. In one patient there was no overlap in the cycle length of the ventricular and supraventricular tachycardias. The inappropriate intervention occurred at a cycle length of 320 ms. Programming of a ventricular tachycardia detection window below $320 \mathrm{~ms}$ could, however, result in the absence of detection of a true ventricular tachycardia with a rather short cycle length.

Sudden onset algorithms attempt to discriminate sinus tachycardia from ventricular tachycardia by rejecting tachycardias in which the rate increases gradually. The sudden onset criterion is programmed in combination with the sustained rate duration, which will overrule the sudden onset criterion if the heart rate remains high. ${ }^{8}$ Our three patients with inappropriate interventions because of sinus tachycardia also had appropriate interventions for ventricular arrhythmias, which were often exercise induced. Therefore the sudden onset criterion resulted in the inappropriate inhibition of the ICD during ventricular tachycardia. This limitation could be overcome if a sustained rate duration had been programmed. However, this would overrule the appropriate inhibition of the ICD during prolonged episodes of sinus tachycardia, which are likely to occur in these young patients. This hypothesis is supported by Brugada and colleagues, who observed that the sustained rate duration override increased sensitivity for ventricular tachycardia detection to $100 \%$ but also increased inappropriate treatment of supraventricular tachycardia from $4 \%$ to $18 \%$. $^{9}$

Stability algorithms attempt to discriminate ventricular tachycardia from atrial fibrillation by rejecting irregular arrhythmias that fulfil rate criteria and they have been shown to be very specific for rejecting atrial fibrillation with rates of less than 170 beats $/ \mathrm{min} .{ }^{8}$ In our patient the ventricular rate during atrial fibrillation was so rapid (231 beats/min) that it fell in the ventricular fibrillation window.

Other detection enhancement algorithms are based on ventricular electrogram morphology analysis, but experience in the clinical setting is limited. Dual chamber detection algorithms use the atrial and the ventricular timing data to discriminate ventricular tachycardia from su- praventricular tachycardia. To date, dual chamber and single chamber detection enhancements have not been compared prospectively. However, it is likely that they will improve discrimination of sinus tachycardia from ventricular tachycardia and may therefore be of benefit in a young population with arrhythmogenic right ventricular dysplasia. ${ }^{10}$

\section{CONCLUSIONS}

Patients with arrhythmogenic right ventricular dysplasia presenting with ventricular fibrillation or haemodynamically unstable ventricular tachycardia have a high recurrence rate requiring appropriate ICD interventions. Unfortunately they often suffer from inappropriate ICD intervention, especially with sinus tachycardia and atrial fibrillation. Programming of the ICD is difficult because the ventricular arrhythmias and supraventricular arrhythmias have overlapping cycle lengths. Furthermore, the ventricular arrhythmias are often exercise induced. These patients may benefit from an ICD with dual chamber detection algorithms.

1 Wichter T, Borggreffe M, Hawerkamp W, et al. Efficacy of antiarrhythmic drugs in patients with arrhythmogenic right ventricular disease. Circulation 1992;86:29-37.

2 Breithard G, Wichter T, Haverkamp W, et al. Implantable cardioverter defibrillator therapy in patients with arrhythmogenic right ventricular cardiomyopathy, long QT syndrome, or no structural heart disease. Am Heart $\mathscr{f} 1994$; 127:1151-8.

3 Link MS, Wang PJ, Haugh CJ, et al. Arrhythmogenic right ventricular dysplasia: clinical results with implantable
cardioverter defibrillators $\mathcal{f}$ Intervent Card Electrophysiol cardioverter

4 Fontaine G, Tonet J, Frank R. Ventricular tachycardia in arrhythmogenic right ventricular dysplasia. In: Zipes D, Jalife J. Cardiac electrophysiology. From cell to bedside, 3rd ed. Philadelphia: WB Saunders, 2000:546-55.

5 McKenna WJ, Thiene G, Nava A, et al. Diagnosis of arrhythmogenic right ventricular dysplasia/ cardiomyopathy. Br Heart f 1994;71:218-18.

6 Pinamonti B, DiLenarda A, Sinagra G, et al. Long term evolution of right ventricular dysplasia/cardiomyopathy. Am Heart f 1995;129:412-15.

7 Tavernier R, Nitzsche R, Jordaens L. What are the expected clinical benefits of the DDD implantable defibrillator. In: Raviele A, ed. Cardiac arrhythmias 1997. Berlin: Springer, 1997:33-6.

8 Swerdlow C, Chen P, Kass R, et al. Discrimination of ventricular tachycardia from sinus tachycardia and atrial fibrillation in tiered-therapy cardioverter-defibrillator. $f$ Am Coll Cardiol 1994;23:1342-55.

9 Brugada J, Mont M, Figueiredo M, et al. Enhanced detection criteria in implantable defibrillators. $\mathcal{F}$ Cardiovasc Electrophysiol 1998;9:261-8.

10 Sadoul N, Henry C, on behalf of the Defender Investigator Group. Long term performance of a dual chamber detection algorithm, the PARAD algorithm, programmed with nominal settings in patients with implantable cardioverter defibrillators [abstract]. PACE 1999;22:125. 